\title{
Optimization of Multimode Fibers for Surface Plasmon Resonance based Sensors under Spectral and Single Wavelength Intensity Interrogation
}

Shivani Sital ( $\square$ shivanisital18@gmail.com )

University of Delhi - South Campus https://orcid.org/0000-0001-5073-3331

\section{Anjli Baliyan}

University of Delhi - South Campus

Enakshi Khular Sharma

University of Delhi - South Campus

Mridula Gupta

University of Delhi - South Campus

\section{Research Article}

Keywords: Surface Plasmon Resonance Sensor, Optical fiber sensor, Wavelength interrogation, Intensity interrogation, Numerical aperture

Posted Date: July 15th, 2021

DOl: https://doi.org/10.21203/rs.3.rs-681813/v1

License: (c) (i) This work is licensed under a Creative Commons Attribution 4.0 International License. Read Full License

Version of Record: A version of this preprint was published at Plasmonics on October 19th, 2021. See the published version at https://doi.org/10.1007/s11468-021-01556-w. 
Optimization of Multimode Fibers for Surface Plasmon Resonance based Sensors under Spectral and Single Wavelength Intensity Interrogation

\author{
Shivani Sital*, Anjli Baliyan, Enakshi Khular Sharma and Mridula Gupta \\ Department of Electronic Science \\ University of Delhi South Campus \\ New Delhi-110021, India
}

E-mail: shivanisital18@gmail.com, anjlibaliyan@gmail.com, enakshi54@yahoo.co.in, mridula@south.du.ac.in

*Corresponding author : shivanisital18@gmail.com

\title{
ACKNOWLEDGMENT
}

The authors would like to thank Council of Scientific and Industrial Research (CSIR), Ministry of Science and Technology, Government of India for the financial support. 


\begin{abstract}
In an optical fiber based SPR sensor, a segment of metal clad silica fiber is used as the sensing element and resonant coupling occurs to the surface plasmon mode excited at the interface of the metal and ambient which surrounds the sensing element. When polychromatic light is transmitted through the sensing element the spectrum of light detected at the other end shows minima at a resonance wavelength. In wavelength interrogation the shift in the resonance wavelength is a measure of change in the refractive index of the ambient. Alternatively, intensity interrogation can be used in which the change in transmitted power at a given wavelength is a measure of change in refractive index of the ambient. The sensitivity and detection accuracy of the sensor depend on the numerical aperture (NA) of the multimode optical fiber used to fabricate the sensor. We have carried out a study of the dependence of the sensitivity and detection accuracy on numerical aperture to obtain the optimal value for a multimode optical fiber which is used to fabricate the sensing element under both wavelength and intensity interrogation. Experimental results on a sensing element fabricated with gold coating on the core of a plastic clad silica fiber are also reported.
\end{abstract}

Keywords - Surface Plasmon Resonance Sensor, Optical fiber sensor, Wavelength interrogation, Intensity interrogation, Numerical aperture 


\section{Introduction}

A metal dielectric interface supports a TM surface wave, also known as surface plasmon mode with the propagation constant a sensitive function of the refractive index of the dielectric layer. This property along with use of dielectric layers in which the refractive index changes due to chemical or biological interactions has resulted in the development of various gas, chemical and bio-molecule sensors [1-4]. These sensors are based on resonant excitation of the surface plasmon waves and are known as surface plasmon resonance (SPR) sensors. Traditionally SPR was measured in the prism coupling Kretschmann configuration [5-8] in which a high index prism coated with a thin metal layer on its base is immersed in the dielectric to be sensed as shown in Fig. 1(a). When monochromatic TM polarized light is incident through the prism on the metal surface and the reflected light measured as a function of angle of incidence, an SPR curve of the type shown in Fig. 1(b) is obtained; the angle at which the minima occurs is the measure of the refractive index $\left(n_{d}\right)$ of the sensor layer and corresponds to the resonance angle at which there is maximum coupling to the metal-dielectric surface plasmon mode. Alternatively, a polychromatic source can be used and reflectance measured for a given angle of incidence to obtain SPR curves of the type shown in Fig. 1(c). The resonance wavelength shifts with change in the refractive index of the dielectric layer. However, both these configurations use bulk-optics and hence form a relatively large system.

(a)
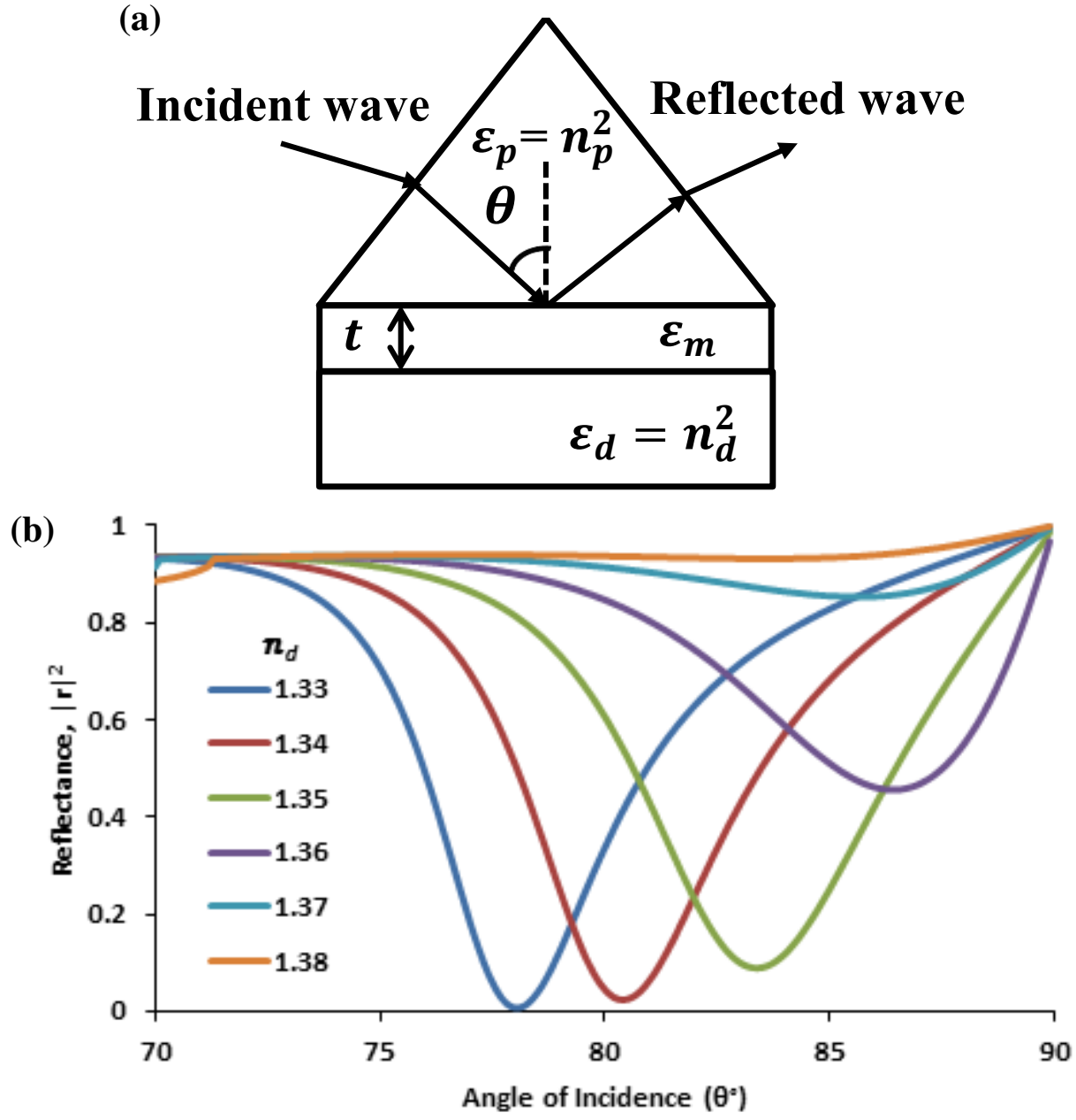


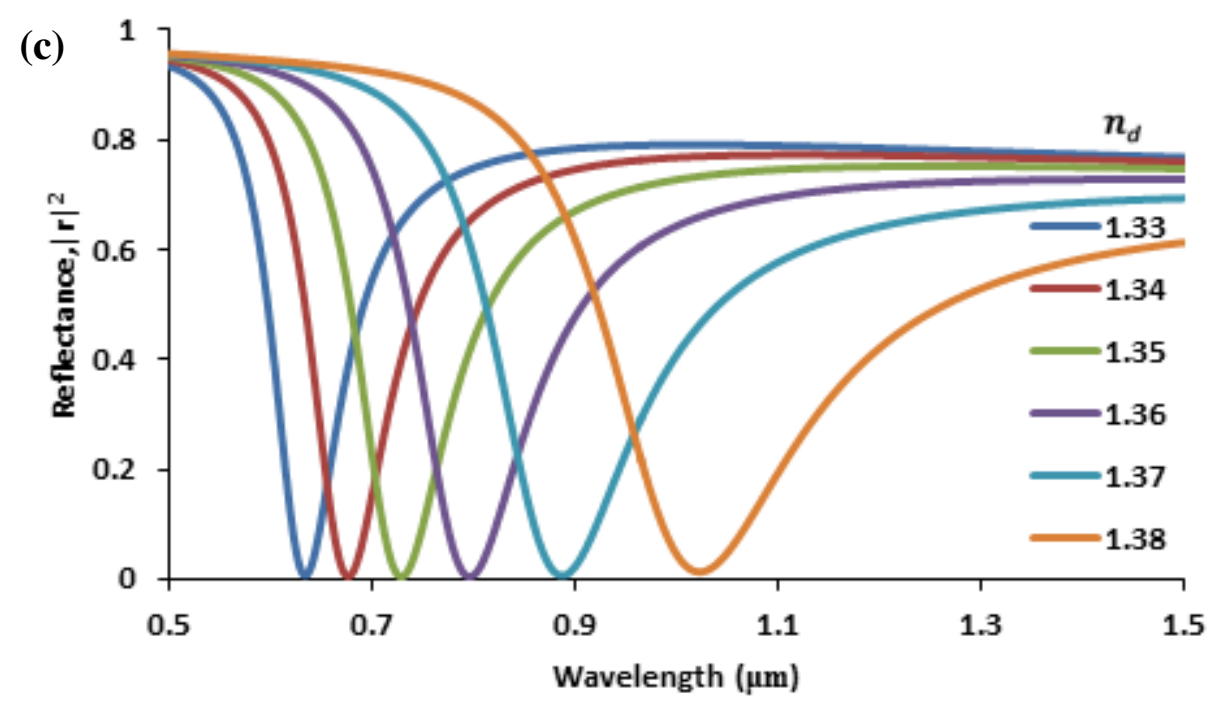

Fig. 1(a) The prism coupling configuration, (b) Reflectance as a function of angle of incidence for different refractive index of ambient $\left(n_{d}\right), \varepsilon_{p}\left(\mathrm{SiO}_{2}\right)=2.1121, \varepsilon_{m}(\mathrm{Au})=-13.082-\mathrm{j} 0.997$ and $d=50 \mathrm{~nm}$ at $\lambda=633 \mathrm{~nm},(\mathrm{c})$ Reflectance as a function of wavelength at angle of incidence $\theta=78^{\circ}$ for different refractive indices of the ambient

Over the last decade considerable interest has grown in the use of SPR based fiber optic sensors [9]. In a fiber based sensor, a segment of metal clad silica fiber is used as the sensing element and resonant coupling occurs to the surface plasmon mode excited at the metal dielectric (ambient) interface as shown in Fig. 2(a). Such sensors are light in weight, rugged and allow remote sensing. Unlike the Kretschmann configuration, all angles of incidence which are allowed by the numerical aperture (NA) of the coupling fiber are possible on the metal interface. For example, for a NA of 0.2 , the allowed range of angles of incidence is from $82^{\circ}$ to $90^{\circ}$. The reflectance as a function of wavelength for this range of angles of incidence for $n_{d}=1.33$ and $n_{d}=1.38$ is shown in Fig. 2(b). Within the angular range resonant coupling occurs at wavelengths varying from $500 \mathrm{~nm}$ to $1100 \mathrm{~nm}$, depending on the refractive index of the dielectric ambient layer. Hence, in fiber optic sensors, polychromatic light is coupled into the fiber and the spectrum of the transmitted light shows a resonance dip which is the signature of the refractive index of the ambient layer as shown in Fig. 2(c). The shift in resonance wavelength is used as the sensing parameter and hence the method is referred to as wavelength interrogation mode. Alternatively, fiber sensor can also be used at a single wavelength in intensity interrogation mode. For example at $\lambda=767 \mathrm{~nm}$ [marked on Fig. 2(b)] the normalized transmitted power shows a continuous change with change in refractive index of the ambient. The sensitivity and the detection accuracy of the sensor are strongly dependent on the NA or allowed range of angles of incidence of the coupling fiber. In this paper we have studied the effect of the numerical aperture for wavelength and single wavelength intensity interrogation mode of the fiber optic sensor and obtained the optimal values. 
(a)

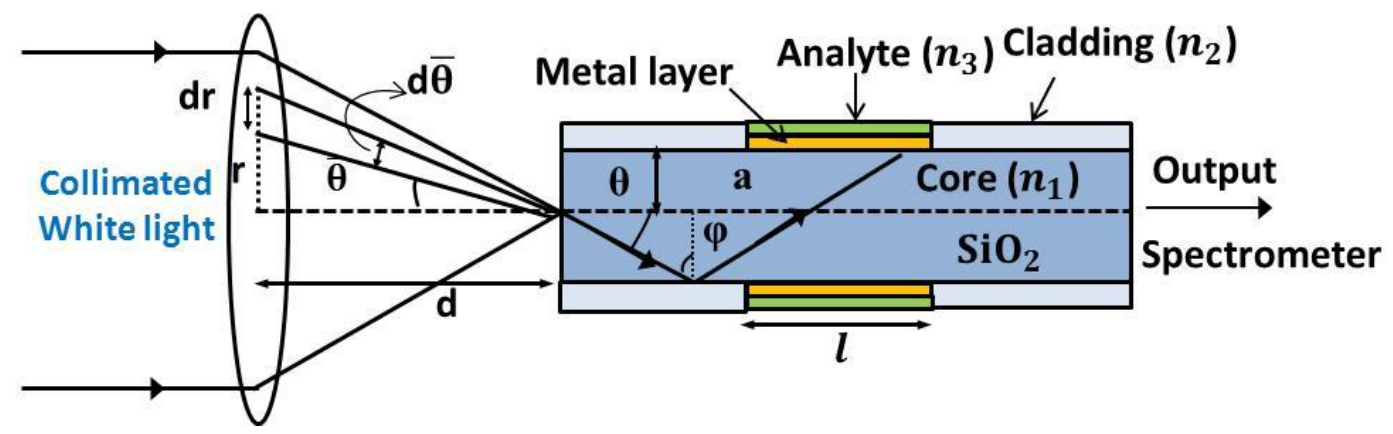

(b)

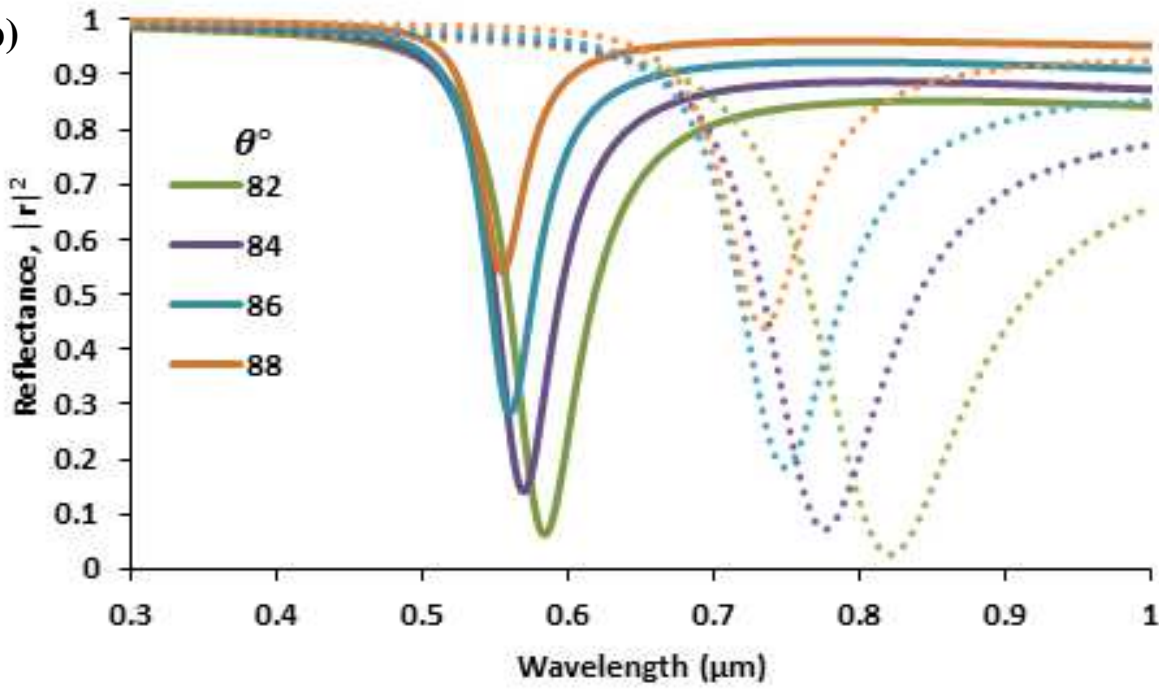

(c)

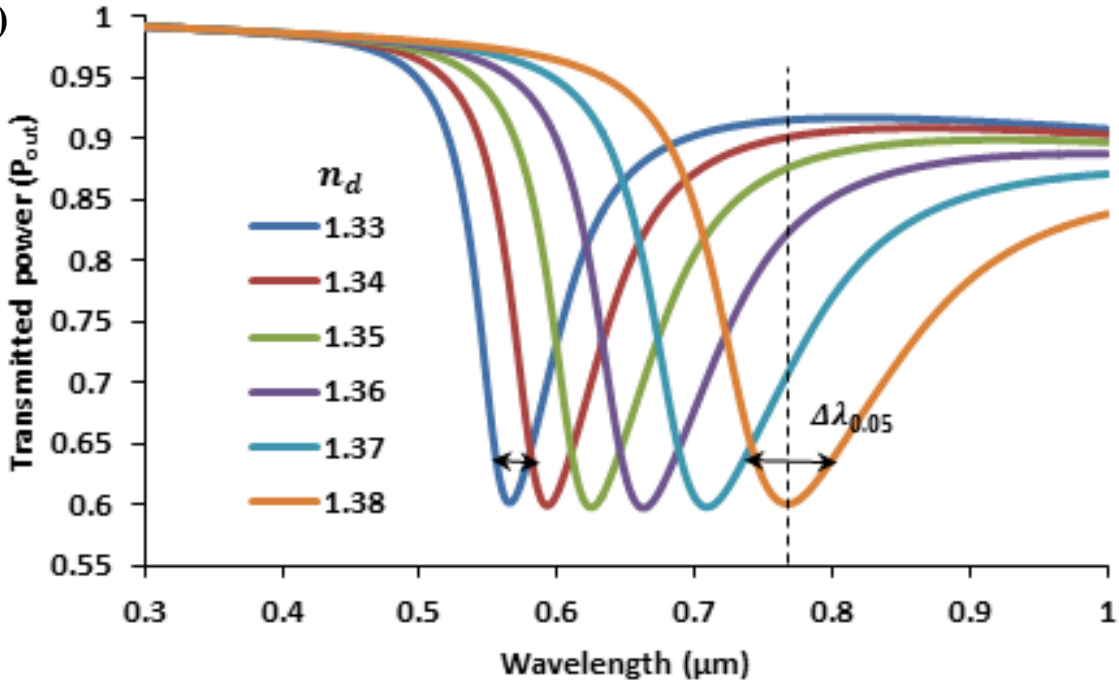

Fig. 2 (a) Coupling arrangement for the excitation of the surface plasmon wave in the metal clad sensing element of the optical fiber, (b) Reflectance as a function of wavelength at different angles of incidence for $n_{d}=$ 1.33 (solid line) and 1.38 (dotted line), (c) Transmittance curves for the SPR based fiber showing shift in resonance wavelength with change in ambient refractive index. The plastic clad multimode fiber is assumed to have $N A=0.2$ with $l / a$ ratio of the sensing element $l / a=33.33$ 
Firstly, we briefly discuss the surface plasmon wave and its excitation in Kretschmann configuration and then present a detailed analysis in the optical fiber configuration to be used in both wavelength and intensity interrogation modes. Finally, a fiber optic SPR sensor has been fabricated and transmittance curves measured with varied refractive indices of the ambient. A satisfactory comparison of shift in resonance wavelength with theoretical calculations is obtained for appropriate choice of the sensor parameters.

\section{Surface Plasmon Mode and Kretschmann Configuration}

As mentioned above, a metal-dielectric interface can support a surface plasmon mode propagating along the surface with the field decaying evanescently into both the metal and dielectric. The effective index of the mode is given by

$$
n_{e}=\sqrt{\frac{\varepsilon_{m} \varepsilon_{d}}{\varepsilon_{m}+\varepsilon_{d}}}
$$

where $\varepsilon_{m}$ and $\varepsilon_{d}$ are the dielectric constant of the metal and the dielectric layer. In a typical metal, the dielectric constant can be written as $\varepsilon_{m}=\varepsilon_{m r}-j \varepsilon_{m i}$, where $\varepsilon_{m r}$ is a negative real part and $\varepsilon_{m i}$ is an imaginary part. The dielectric constant for gold can be evaluated from the extensively used Drude model [6] as

$$
\varepsilon_{m}(\lambda)=\varepsilon_{m r}-j \varepsilon_{m i}=1-\frac{\lambda^{2} \lambda_{c}}{\lambda_{p}^{2}\left(\lambda_{c}-j \lambda\right)}
$$

with $\lambda_{p}=0.16826 \mu \mathrm{m}$ as the plasma wavelength and $\lambda_{c}=8.9342 \mu \mathrm{m}$ as the collision wavelength.

For the Kretschmann configuration, a gold metal layer of thickness $t=50 \mathrm{~nm}$ can be deposited on a prism made of fused silica with dielectric constant $\varepsilon_{p}=n_{p}^{2}$ given by Sellmeier dispersion relation [10] as

$$
n_{p}(\lambda)=\sqrt{1+\frac{a_{1} \lambda^{2}}{\lambda^{2}-b_{1}}+\frac{a_{2} \lambda^{2}}{\lambda^{2}-b_{2}}+\frac{a_{3} \lambda^{2}}{\lambda^{2}-b_{3}}}
$$

with the Sellmeier coefficients given by $a_{1}=0.6961663, a_{2}=0.4079426, a_{3}=0.8974794, b_{1}=0.004679148$, $b_{2}=0.01351206$ and $b_{3}=97.93400$.

When monochromatic light is incident at an angle $\theta$, the reflectance $R=|r|^{2}$ as a function of angle of incidence $\theta$ can be evaluated by

$$
r=\frac{r_{p m}+r_{m d} e^{-2 j \kappa_{2} t}}{1+r_{p m} r_{m d} e^{-2 j \kappa_{2} t}}
$$

where

$$
r_{a b}=\frac{\varepsilon_{b} \kappa_{a}-\varepsilon_{a} \kappa_{b}}{\varepsilon_{b} \kappa_{a}+\varepsilon_{a} \kappa_{b}}, \kappa_{a}=k_{0} \sqrt{\varepsilon_{a}-\varepsilon_{3} \sin ^{2} \theta}
$$

and subscripts $a$ and $b$ are $p, m$ or $d$ corresponding to the prism, metal and ambient respectively.

The SPR curves are obtained by plotting reflectance as a function of angle of incidence for different refractive indices of the sensing layer at a given wavelength. For the He-Ne laser, the wavelength $\lambda=633 \mathrm{~nm}, \varepsilon_{m}(\mathrm{Au})=-$ 13.0822-j0.9977, $\varepsilon_{p}=2.1121$ and the corresponding SPR curves are shown in Fig. 1(b). In this angle 
interrogation mode, the angle at which the reflectance is a minimum corresponds to the situation when the evanescent wave at total internal reflection is phase matched with the propagating surface plasmon wave or

$$
n_{p} \sin \theta_{\min }=n_{e} \simeq \sqrt{\frac{\varepsilon_{m r} \varepsilon_{d}}{\varepsilon_{m r}+\varepsilon_{d}}}
$$

More correctly, $n_{e}$ corresponds to the effective index of a leaky mode at the metal-dielectric interface, although the difference for adequately thick metal layers is very small [11].

The Kretschmann configuration can also be used in wavelength interrogation mode, i.e., using a polychromatic source, keeping angle of incidence constant. The plot of reflectance versus wavelength now forms the SPR curves, as shown in Fig. 1(c) at $\theta=78^{\circ}$ for different refractive indices of the dielectric layer. The fiber optic SPR sensor uses a similar wavelength interrogation as discussed in the next section.

\section{The Optical Fiber SPR Sensor and Numerical Aperture}

In a fiber optic SPR sensor, the cladding of a plastic clad multimode fiber is removed in mid-length and the silica core with index $n_{1}$ is coated with a thin layer of gold $(t=50 \mathrm{~nm})$ to form the sensing element. Fig.2(a) shows the schematic diagram of coupling arrangement for the excitation of the surface plasmon waves in a metal clad sensing element of the optical fiber. Collimated light from a source is focused at the center of one end of the plastic clad fiber and the transmitted light is detected at the other end of the fiber. The change in the refractive index $n_{d}$ of the ambient which surrounds the sensing element is determined by observing the change in the spectrum in wavelength interrogation method or intensity in the single wavelength interrogation method. Assuming that all the guided rays are launched into the fiber using a collimated beam of intensity $I_{0}$ and a microscope objective, the power, $d P(\bar{\theta})$ launched between angle $\bar{\theta}$ and $\bar{\theta}+d \bar{\theta}$ at the input is given by $d P(\bar{\theta})=$ $I_{0} 2 \pi d^{2}\left(\tan \bar{\theta} / \cos ^{2} \bar{\theta}\right) d \bar{\theta}$ and can be related by Snell's law to the power distribution in rays [12, 13] incident at an angle between $\varphi$ and $\varphi+d \varphi$ on the silica-metal surface as

$$
d P(\varphi)=I_{0} 2 \pi d^{2} \frac{n_{1}^{2} \cos \varphi \sin \varphi}{\left(1-n_{1}^{2} \cos ^{2} \varphi\right)^{2}} d \varphi
$$

The number of reflections, $N_{\text {ref }}(\varphi)$, in the sensing region is a function of angle $\varphi$, as well as the ratio of length of the sensing region, $l$ and diameter of the fiber $2 a$ given by

$$
N_{\text {ref }}=\frac{l}{2 a} \cot \varphi
$$

In order to determine the transmitted power, the reflectance for TM polarization for a single reflection is raised to the power of the number of reflections at the metal-dielectric interface at the specific propagating angle. The TE polarization does not excite the surface wave hence the reflection coefficient is equal to unity. Therefore, for an un-polarized collimated light source, the generalized expression for the normalized transmitted power, $P_{\text {out }}(l)$, in an SPR based fiber optic sensor is given by 


$$
P_{\text {out }}(l)=\frac{1}{2}\left[\frac{\int_{\varphi_{c} / 2}^{\pi / 2} \frac{n_{1}^{2} \cos \varphi \sin \varphi}{\left(1-n_{1}^{2} \cos ^{2} \varphi\right)^{2}}\left(|r|^{2}\right)^{N_{r e f}} d \varphi}{\int_{\varphi_{c} / 2}^{\pi / 2} \frac{n_{1}^{2} \cos \varphi \sin \varphi}{\left(1-n_{1}^{2} \cos ^{2} \varphi\right)^{2}} d \varphi}+1\right]
$$

where $\varphi_{c}$ is the critical angle at the silica-plastic clad interface. In writing this expression it is assumed that light incident on the metal-dielectric interface has equal fraction of TE and TM-polarization. The critical angle $\varphi_{c}$ is determined by the numerical aperture of the plastic clad fiber given by $N A=\sqrt{n_{1}^{2}-n_{c l}^{2}}$ where $n_{c l}$ is refractive index of the plastic cladding and $\varphi_{c}$ can be shown to be given by $\varphi_{c}=\cos ^{-1}\left(N A / n_{1}\right)$.

\subsection{Wavelength Interrogation}

The SPR curves are obtained by plotting the normalized transmitted power as a function of wavelength and are shown in Fig. 2(c) corresponding to a sensing element with $l / a=33.33$ in a plastic clad fiber of $N A=0.2\left(\varphi_{c}=\right.$ $82^{\circ}$ ). The curves show a minima at the resonance wavelength which shifts with the change in the refractive index of the ambient. The larger is the shift of the resonance wavelength $\Delta \lambda_{r}$ for a given change in refractive index $\Delta n_{d}$, the higher is the sensitivity of the sensor defined as $S_{n}=\Delta \lambda_{r} / \Delta n_{d}$ in nm/RIU. Another figure of merit is detection accuracy $(D A)$ which is related to the sharpness of the resonance dip. The narrower the resonance dip the higher is the detection accuracy. Detection accuracy can be well defined as the inverse of the spectral width at a transmittance 5\% higher than minima [1] [as marked in Fig. 2(c)]

$$
D A=\frac{1}{\Delta \lambda_{0.05}}
$$

For example, the fiber of $N A=0.2$ shows a sensitivity of $4040 \mathrm{~nm} / \mathrm{RIU}$ and detection accuracy varies from 20 $\mu \mathrm{m}^{-1}$ to $48 \mu \mathrm{m}^{-1}$ for $l / a=33.33$ in the range of ambient refractive index 1.33 to 1.38 .

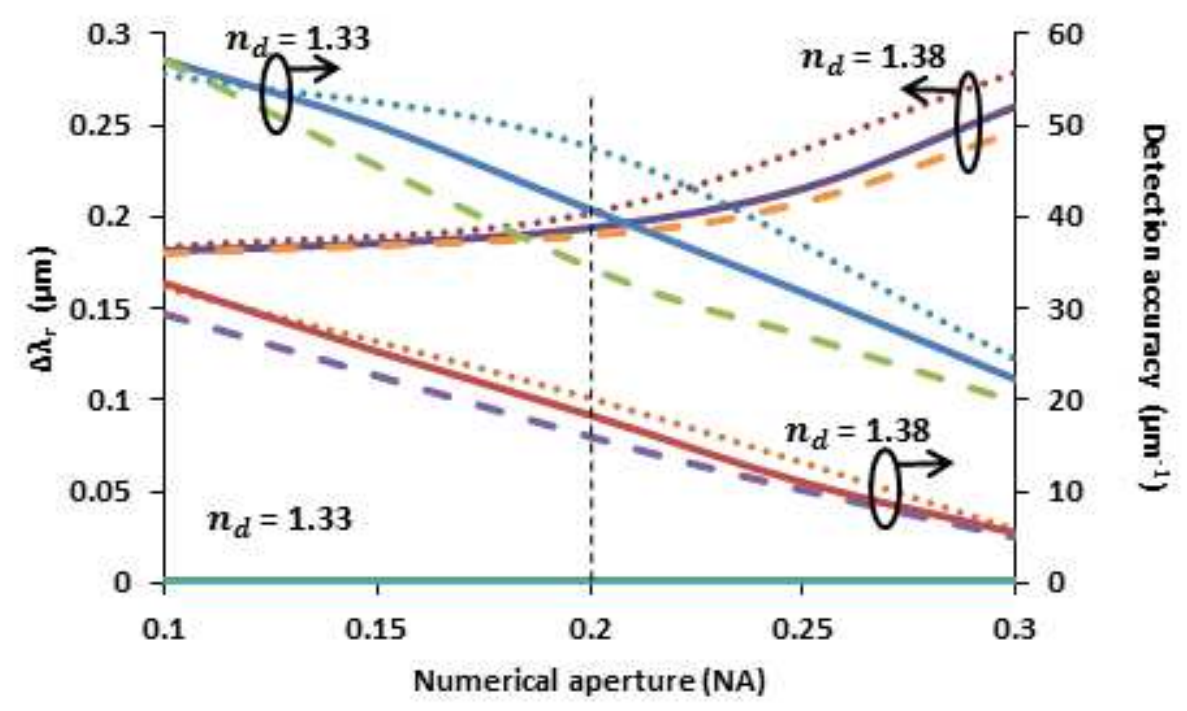

Fig. 3 Change in shift in resonance wavelength, $\Delta \lambda_{r}$, on primary y-axis and detection accuracy on secondary yaxis with NA of the sensing fiber for refractive index, $n_{d}=1.33$ and 1.38 for three $l / a$ ratios of 33.33 (dotted line), 66.66 (solid line) and 100 (dashed line) 
To study the effect of $N A$ and $l / a$ ratio on the sensitivity and detection accuracy, we carried out calculations of the SPR curves corresponding to different values of numerical aperture and refractive indices of the ambient. The shift in resonance wavelength with respect to resonance wavelength at $n_{d}=1.33, \Delta \lambda_{r}$, is plotted for $n_{d}=$ 1.38 in Fig. 3 along with the detection accuracy for $n_{d}=1.33$ and $n_{d}=1.38$ as a function of numerical aperture for three values of $l / a$ ratio. The curves show that although for high numerical apertures the shift in resonance wavelength is large resulting in high sensitivity, the corresponding detection accuracy decreases. Similarly for low numerical apertures the shift in resonance wavelength is lower, while the detection accuracy is high. Hence, an optimal value needs to be chosen. For an optical fiber of $N A=0.2$, the detection accuracy and shift in resonance wavelength are both satisfactory and hence for an $N A=0.2$ can be considered to be optimal for wavelength interrogation. The corresponding SPR curves are as shown in Fig. 2(c); shifts in resonance wavelength and detection accuracy with the change in refractive index of the sensing layer are plotted in Fig. 4. The sensitivity is obtained as $4040 \mathrm{~nm} / \mathrm{RIU}$ and detection accuracy lies between $20 \mu \mathrm{m}^{-1}$ to $48 \mu \mathrm{m}^{-1}$ in the range of refractive index of ambient from 1.33 to 1.38 .

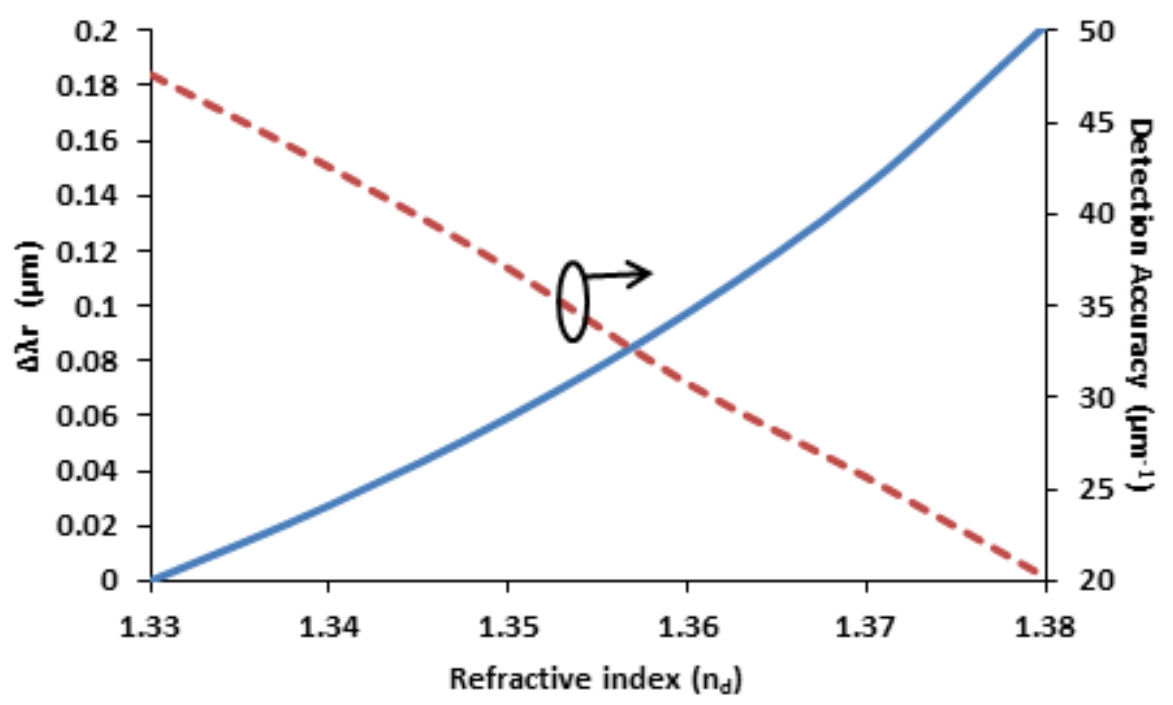

Fig. 4 Change in shift in resonance wavelength, $\Delta \lambda_{r}$, on primary y-axis (solid line) and detection accuracy, $D A$, on secondary y-axis (dashed line) with refractive index of the ambient for the SPR based fiber optic sensor with fiber of $N A=0.2$ and $l / a$ ratio of 33.33

\subsection{Intensity Interrogation}

As mentioned above, the multimode plastic clad fiber sensor can also be used at a single wavelength in intensity interrogation mode. For the optical fiber of $N A=0.2$, the SPR curves plotted in Fig. 2(b) show that at a chosen wavelength of $\lambda=767 \mathrm{~nm}$ the normalized transmitted power continuously increases with change in refractive index from 1.33 to 1.38 . Hence, the sensor of numerical aperture 0.2 can be used in the intensity interrogation at wavelength $\lambda=767 \mathrm{~nm}$. The plot of transmitted power versus $N A$ as a function of refractive index of the ambient also shows that $N A=0.2$ is the appropriate value as shown in Fig. 5(a). 

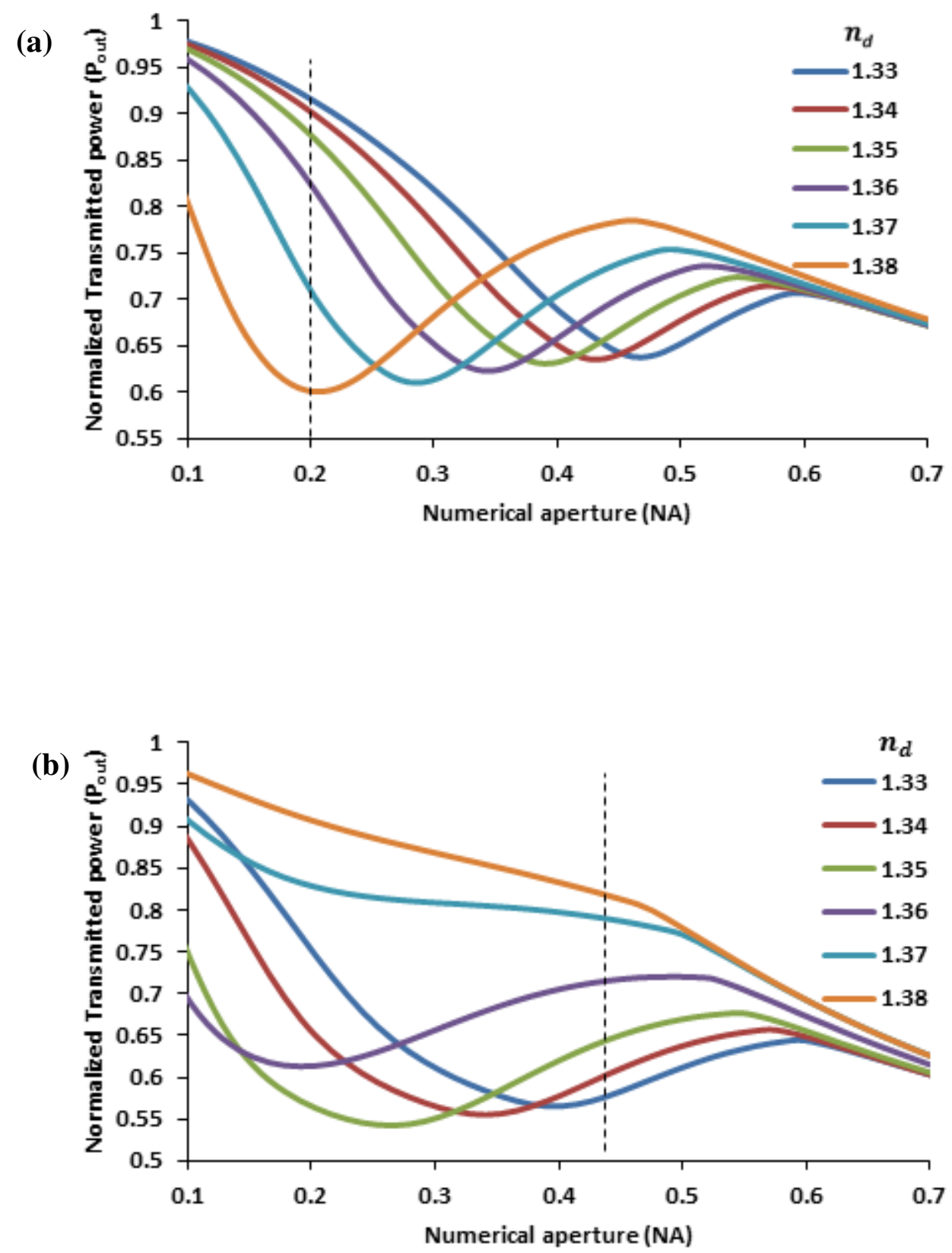


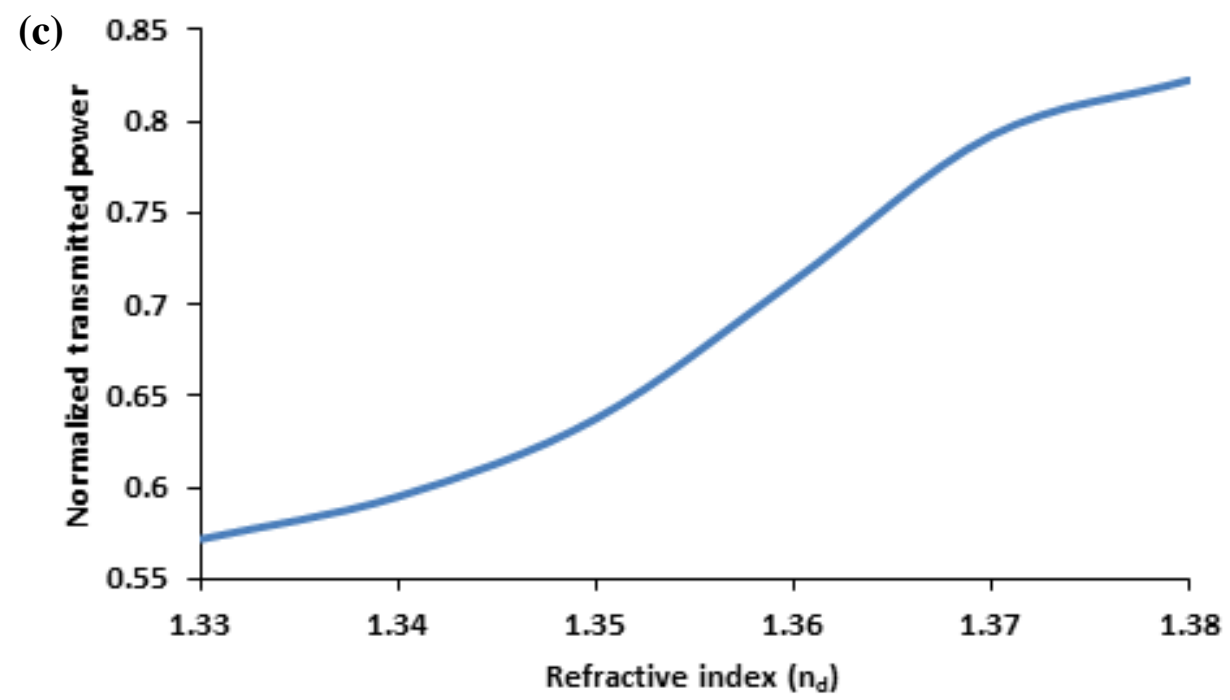

Fig. 5 Normalized transmitted power for the SPR based sensor for numerical aperture with $l / a$ ratio $=$ 33.33. The optimal numerical aperture for single spectral interrogation is also shown by the dashed line for (a) $\lambda=767 \mathrm{~nm}$, (b) $\lambda=633 \mathrm{~nm}$, (c) Variation of normalized transmitted power with refractive index of the ambient for the SPR based fiber optic sensor with fiber of $N A=0.42$ and $\lambda=633 \mathrm{~nm}$

We also looked at the possible use of the single wavelength intensity interrogation mode at the He-Ne laser wavelength of $633 \mathrm{~nm}$. The transmitted power as a function of numerical aperture of the sensor for $\lambda=633 \mathrm{~nm}$, plotted in Fig. 5(b), shows that for numerical aperture of 0.42 there is continuous and significant change of transmitted power on increasing the refractive index of the sensing medium. The normalized transmitted power keeps on increasing with increase in refractive index of the sensing medium as shown in Fig. 5(c). Hence, for He-Ne Laser wavelength $(633 \mathrm{~nm})$, the fiber optic sensor of numerical aperture 0.42 is the optimum to be used in the intensity interrogation mode.

\section{Experimental results}

The spectral interrogation was also carried out experimentally by fabricating a sensing element on an available multimode plastic clad fiber with a silica core of radius $300 \mu \mathrm{m}$. The plastic cladding was removed from the middle section over a length of $3 \mathrm{~cm}$ and the unclad portion was coated with a thin layer of chromium and a gold metal layer by thermal evaporation technique. A chromium layer of 2-3 nm is essential for the adhesion of gold. Light from a polychromatic source (AvaLight-HAL) is focused at the input of the multimode plastic clad fiber using a microscope objective and the transmitted spectra is recorded at the other end of the fiber using a spectrometer (Avaspec ULSi 3648). Fig. 6 shows the schematic diagram of the experimental setup. The sensing probe is fixed in a glass flow cell in which sugar solutions (analyte) with varying concentrations (and hence refractive index) can be poured. The refractive indices of the solutions were also measured by Abbe refractometer of accuracy upto third decimal place. The normalized transmission spectra of the fabricated sensor probe measured for different refractive indices of sugar solutions in the range of 1.333 to 1.383 are shown in Fig. 7(a). On increasing the refractive index of the sensing medium the resonance wavelength shifts toward the longer wavelength with sensitivity of $359 \mathrm{~nm} / \mathrm{RIU}$. 


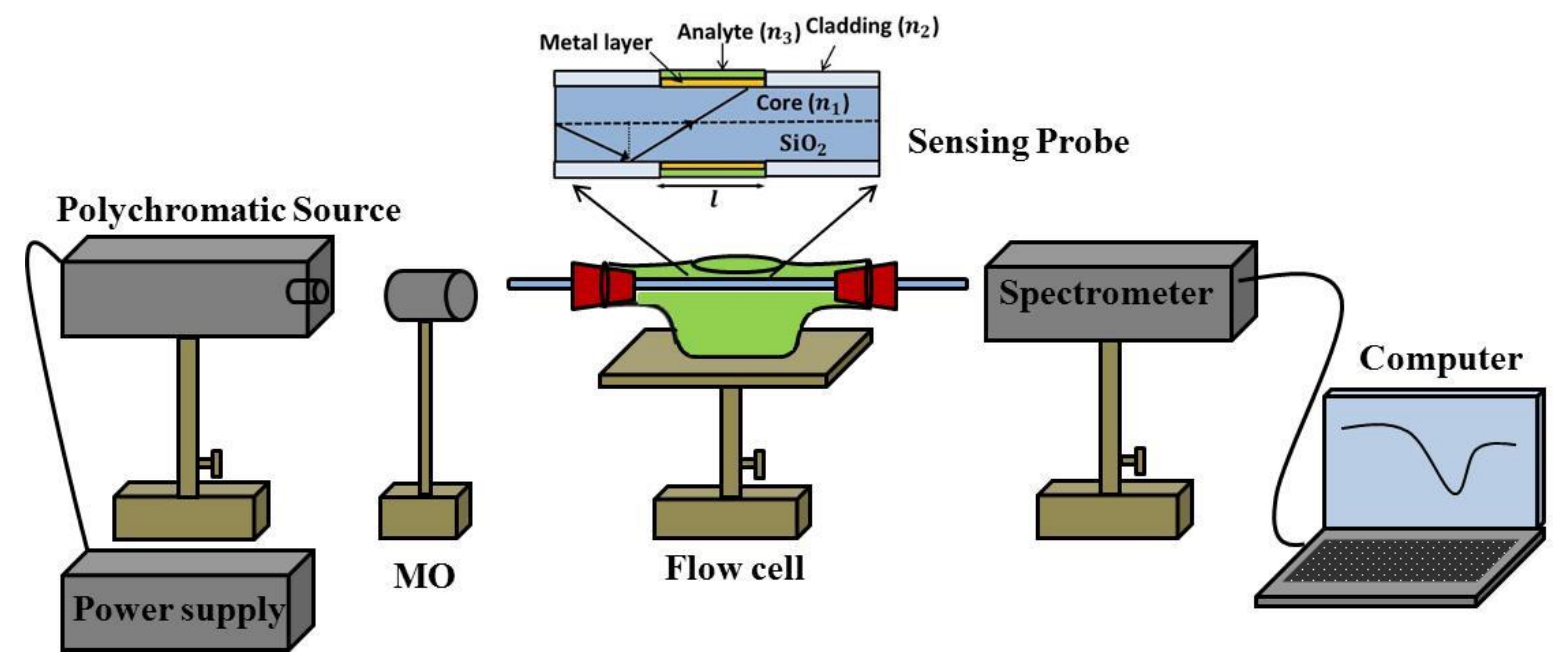

Fig. 6 Experimental setup for fiber optic SPR based sensor
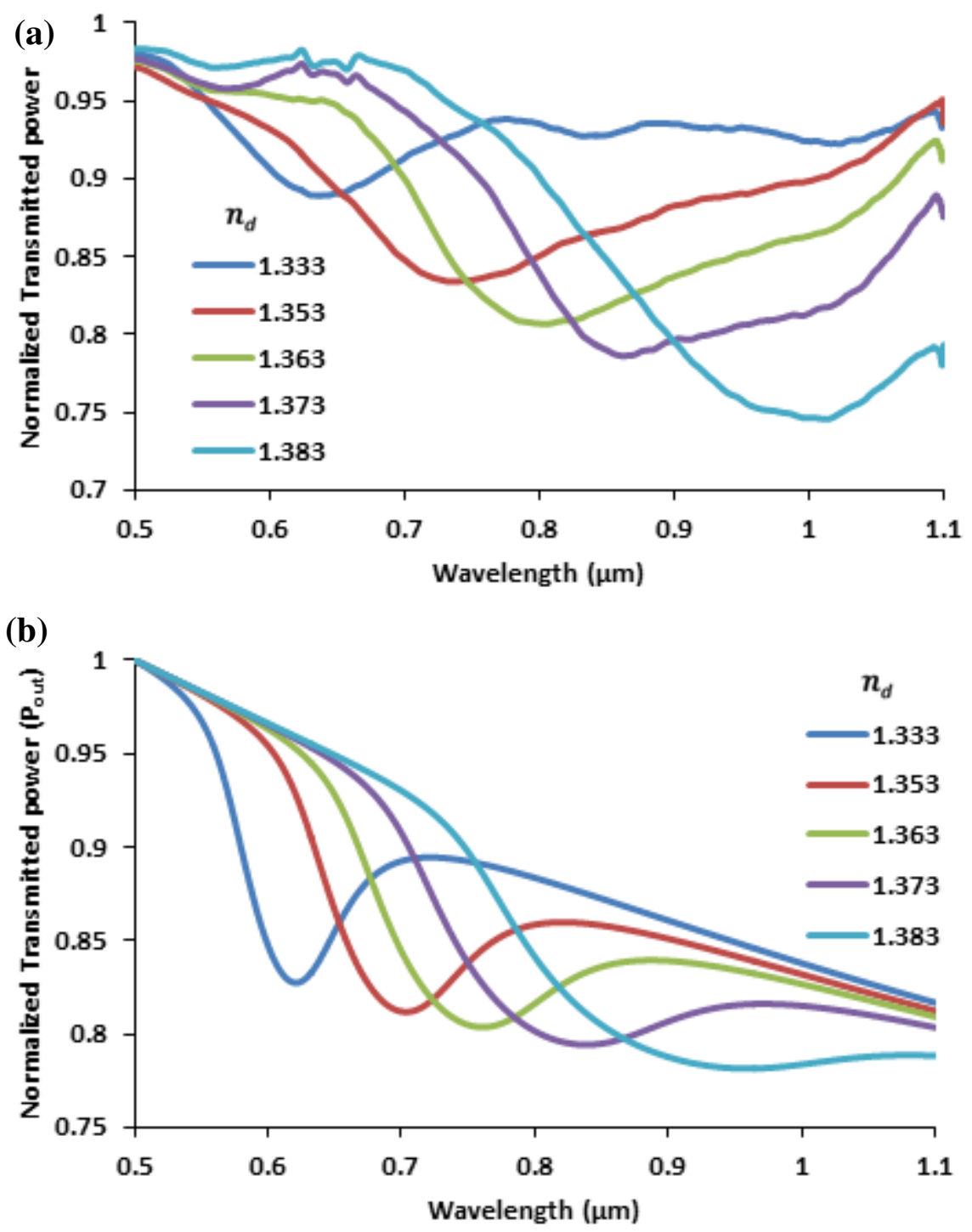
Fig. 7 Normalized transmitted power is plotted as a function of wavelength for different refractive indices for sensing probe with $l / a=100$ (a) Experimental, (b) theoretical model for $N A=0.3$ with metal thickness of 90 $\mathrm{nm}$

To understand the transmittance curves of the fabricated sensor probe fiber theoretically, we considered a silica core of radius $300 \mu \mathrm{m}$ with $l / a=100$. Since the numerical aperture of the fiber and thickness of the gold metal layer of the sensing element were not known accurately, we studied the effect of both simultaneously on transmission spectrum in the range of refractive indices from 1.33 to 1.38 . The choice of numerical aperture 0.3 with thickness of gold metal layer of $90 \mathrm{~nm}$ gives a shift in resonance wavelength which is comparable with the shift obtained experimentally as can be seen from the SPR curves plotted in Fig. 7(b).

\section{Conclusion}

In conclusion, we have presented an analysis for the optimization of numerical aperture of a plastic clad multimode fiber to be used for an SPR based sensor in both spectral interrogation and single wavelength intensity interrogation mode. For a gold clad sensing element with $l / a=33.33$ for the refractive index of the ambient in the range 1.33 to 1.38 the optimized numerical aperture is obtained as $N A=0.2$ for spectral interrogation mode. At this optimal value the sensitivity is estimated to be $4040 \mathrm{~nm} / \mathrm{RIU}$ and detection accuracy lies between $20 \mu^{-1}$ to $48 \mu^{-1}$. In single wavelength intensity interrogation, at the He-Ne laser wavelength $(633 \mathrm{~nm})$ the optimized numerical aperture is obtained as $N A=0.42$. We have also experimentally fabricated a fiber optic SPR sensor and measured the SPR curves. A satisfactory comparison of experimental results to theoretical calculation is obtained by assuming a numerical aperture of 0.3 and gold metal layer thickness $90 \mathrm{~nm}$ for the sensing element with $l / a=100$.

\section{ACKNOWLEDGMENT}

The authors would like to thank Council of Scientific and Industrial Research (CSIR), Ministry of Science and Technology, Government of India for the financial support. 


\section{Declarations}

Funding: The authors would like to thank Council of Scientific and Industrial Research (CSIR), Ministry of Science and Technology, Government of India for the financial support.

\section{Conflicts of interest/Competing interests:}

- The authors have no relevant financial or non-financial interests to disclose.

- The authors have no conflicts of interest to declare that are relevant to the content of this article.

- All authors certify that they have no affiliations with or involvement in any organization or entity with any financial interest or non-financial interest in the subject matter or materials discussed in this manuscript.

- The authors have no financial or proprietary interests in any material discussed in this article.

Availability of data and material: Not Applicable

Code availability: Not Applicable

Authors' contributions : All authors contributed to the study conception and design. Material preparation, data collection and analysis were performed by Shivani Sital and Anjli Baliyan. The first draft of the manuscript was written by Shivani Sital and all authors commented on previous versions of the manuscript. All authors read and approved the final manuscript.

Ethics approval: This is an observational study. Hence, no ethical approval is required.

Consent to participate: Not Applicable

Consent for publication: Not Applicable

Compliance with Ethical Standards:

- Disclosure of potential conflicts of interest -NA

- Research involving Human Participants and/or Animals - NA

- Informed consent -NA 


\section{REFERENCES}

[1] Homola J, Sinclair S, Gauglitz GY (2006) Surface plasmon resonance sensors: review, Sensor and Actuators B, 115, 344-348.

[2] Baliyan A, Bhatia P, Gupta BD, Sharma EK, Kumari A and Gupta R (2013) Surface plasmon resonance based fiber optic sensor for the detection of triacylglycerides using gel entrapment technique, Sensors and Actuators B, 188 917-922.

[3] Bhatia P, Gupta BD (2013) Surface plasmon resonance based fiber optic ammonia sensor utilizing bromocresol purple, Plasmonics, 8, 779-784.

[4] Singh S and Gupta BD (2013) Fabrication and characterization of a surface plasmon resonance based fiber optic sensor using gel entrapment technique for detection of low glucose concentration, Sensors and Actuators B, 177, 589-595.

[5] Kretschmann E and Raether H (1968) Radiative decay of non radiative surface plasmons excited by light, Z. Naturforsh, 23 2135-2136.

[6] Homola J (1997) On the sensitivity of surface plasmon resonance sensors with spectral interrogation, Sensor and Actuator B, 41 207-211.

[7] Gwon HK and Lee SH (2010) Spectral and angular responses of surface plasmon resonance based on the Kretschmann prism configuration, Material Transactions, 51, 1150-1155.

[8] Paliwal A, Gaur R, Sharma A, Tomar M, Gupta V (2016) Sensitive optical biosensor based on surface plasmon resonance using $\mathrm{ZnO} / \mathrm{Au}$ bilayered structure, Optik 127, 7642-7647.

[9] Gupta BD, Srivastava SK, Verma R (2015) Fiber Optics Sensors based on Plasmonics, World Scientific

[10] Adams MJ (1981) An Introduction to Optical Waveguides, John Wiley \& Sons

[11] Sital S, Sharma EK (2014) Excitation of Leaky Surface Plasmon Modes in Sensors, International Conference on Optics and Optoelectronics, ICOL 2014, 5-8 March 2014, IRDE Dehradun, India.

[12] Gupta BD, Sharma A, Singh CD (1993) Evanescent wave absorption sensors based on uniform and tapered fibers, Int. J. Optoelectron, 8, 409.

[13] Gupta BD, Singh CD and Sharma A (1994) Fiber-optic evanescent field absorption sensor: effect of launching condition and the geometry of the sensing region, Optical Engineering 33, 1864-1868. 